PROCEEDINGS OF THE

AMERICAN MATHEMATICAL SOCIETY

Volume 128, Number 7 , Pages $2007-2015$

S 0002-9939(99)05263-6

Article electronically published on November 24, 1999

\title{
GENERATION OF LINEAR EVOLUTION OPERATORS
}

\author{
NAOKI TANAKA \\ (Communicated by Dale Alspach)
}

\begin{abstract}
This paper is devoted to the problem of generation of evolution operators associated with linear evolution equations in a general Banach space. The stability condition is proposed from the viewpoint of finite difference approximations. It is shown that linear evolution operators can be generated even if the stability condition given here is assumed instead of Kato's stability condition.
\end{abstract}

\section{INTRODUCTION}

The theory of abstract linear evolution equations plays a basic role in studying the theory of abstract quasi-linear evolution equations which has been recently developed by Kato 2, and Kobayasi and Sanekata [4. This paper is devoted to the generation of an evolution operator associated with the linear evolution equation

$$
u^{\prime}(t)=A(t) u(t) \quad \text { for } t \in[s, T]
$$

in a general Banach space $X$. Some fundamental and important theorems concerning evolution operators are given by several authors (for example, Kato [1] and Kobayasi [3]). In those articles, the stability condition proposed by Kato [1] is assumed, but it is stated in his paper that this stability is stronger than the usual one used in the theory of finite difference approximations. We are here interested in weakening the stability condition from the viewpoint of finite difference approximations. The purpose of the present paper is to show that Theorem I of [2] remains true even if the stability condition given here (see condition (A1)) is assumed instead of Kato's stability condition. The Main Theorem is given in Section 1.

Here is listed the notation used in this paper. Given Banach spaces $\mathfrak{X}, \mathfrak{Y}, \ldots$ the associated norms are denoted by $\|\cdot\|_{\mathfrak{X}},\|\cdot\|_{\mathfrak{Y}}$ and so on. The symbol $\|\mathfrak{A}\|_{\mathfrak{Y}, \mathfrak{X}}$ denotes the norm of $\mathfrak{A}$ in the Banach space $B(\mathfrak{Y}, \mathfrak{X})$ which consists of all bounded linear operators on $\mathfrak{Y}$ to $\mathfrak{X}$. We write $B(\mathfrak{X})$ for $B(\mathfrak{X}, \mathfrak{X})$ and $\|\mathfrak{A}\|_{\mathfrak{X}}$ for $\|\mathfrak{A}\|_{\mathfrak{X}, \mathfrak{X}}$. The space $C_{*}([0, T]: B(\mathfrak{Y}, \mathfrak{X}))$ consists of all strongly continuous operator functions on $[0, T]$ into $B(\mathfrak{Y}, \mathfrak{X})$. For such functions we use the supremum norm $\|\mid \mathfrak{A}\|_{\mathfrak{Y}, \mathfrak{X}}=$ $\sup \left\{\|\mathfrak{A}(t)\|_{\mathfrak{Y}, \mathfrak{X}}: t \in[0, T]\right\}$.

Received by the editors May 4, 1998 and, in revised form, August 24, 1998.

1991 Mathematics Subject Classification. Primary 47D06; Secondary 34G10.

Key words and phrases. Linear evolution operator, stability condition, intertwining condition.

(C)2000 American Mathematical Society 


\section{Statement of Main Theorem}

Let $X$ and $Y$ be Banach spaces such that $Y$ is densely and continuously embedded in $X$. We begin by setting up basic hypotheses on $A$ appearing in (CP).

(A1) There exist $M \geq 1$ and $\lambda_{0}>0$ such that $\left(I-\left(t_{i}-t_{i-1}\right) A\left(t_{i}\right)\right)^{-1} \in B(X)$ and

$$
\left\|\prod_{i=1}^{k}\left(I-\left(t_{i}-t_{i-1}\right) A\left(t_{i}\right)\right)^{-1}\right\|_{X} \leq M
$$

for every finite sequence $\left\{t_{i}\right\}_{i=0}^{k}$ with $0 \leq t_{0}<t_{1}<\cdots<t_{k} \leq T$ and $t_{i}-t_{i-1} \leq \lambda_{0}$ for $i=1,2, \ldots, k$.

Remark 1. Let $\left\{t_{i}\right\}_{i=0}^{k}$ be a sequence such that $0 \leq t_{0}<t_{1}<\cdots<t_{k} \leq T$, and $x_{0} \in X$. Roughly speaking, condition (A1) means that the difference equation

$$
\left(x_{i}-x_{i-1}\right) /\left(t_{i}-t_{i-1}\right)=A\left(t_{i}\right) x_{i} \quad \text { for } i=1, \ldots, k
$$

has a solution $\left\{x_{i}\right\}_{i=1}^{k}$ satisfying the estimate $\left\|x_{i}\right\|_{X} \leq M\left\|x_{0}\right\|_{X}$ for $i=1, \ldots, k$.

(A2) For $t \in[0, T], D(A(t)) \supset Y . A \in C_{*}([0, T]: B(Y, X))$.

According to Kato's device, we use another Banach space $Z$ and an operator $S \in B(Y, Z)$ such that

$$
\|u\|_{Y}=\|u\|_{X}+\|S u\|_{Z}
$$

for $u \in Y$, and assume that there exists a strongly continuous and nonexpansive homomorphism $\Xi$ of the algebra $B(X)$ into $B(Z)$, in order to explain the "intertwining condition".

Let $t \in(0, T]$ be fixed arbitrarily. Then we have, by (A1), $(I-\lambda A(t))^{-1} \in B(X)$ and $\left\|(I-\lambda A(t))^{-1}\right\|_{X} \leq M$ for $\lambda \in\left(0, \lambda_{0} \wedge t\right]$. Since $Y$ is dense in $X$ we have $\lim _{\lambda \downarrow 0}(I-\lambda A(t))^{-1} x=x$ for every $x \in X$. Set $J(\lambda)=\Xi(I-\lambda A(t))^{-1}$ for $\lambda \epsilon$ $\left(0, \lambda_{0} \wedge t\right]$. By the property of $\Xi$ we have $J(\lambda) \in B(Z)$ and $\lim _{\lambda \downarrow 0} J(\lambda) z=z$ for every $z \in Z$. Moreover, the family $\left\{J(\lambda): \lambda \in\left(0, \lambda_{0} \wedge t\right]\right\}$ satisfies the equation $J(\lambda)=J(\mu)((\mu / \lambda) I+(1-\mu / \lambda) J(\lambda))$ for $\lambda, \mu \in\left(0, \lambda_{0} \wedge t\right]$. Hence there exists a unique family $\{\bar{A}(t): t \in(0, T]\}$ of closed linear operators in $Z$ such that

$$
(I-\lambda \bar{A}(t))^{-1}=\Xi(I-\lambda A(t))^{-1}
$$

for $t \in(0, T]$ and $\lambda \in\left(0, \lambda_{0} \wedge t\right]$. The intertwining condition is stated as follows:

(A3) There exists $B \in C_{*}([0, T]: B(Z))$ such that

$$
S(I-(t-s) A(t))^{-1} \supset(I-(t-s)(\bar{A}(t)+B(t)))^{-1} S
$$

for $0 \leq s<t \leq T$ and $t-s \leq \varepsilon_{0}:=\lambda_{0} \wedge\left(1 / 2 M\left|\|B \mid\|_{Z}\right)\right.$.

Throughout this paper we assume conditions (A1) through (A3). The purpose of this paper is to prove the following theorem.

Main Theorem. There exists a unique family $\{U(t, s):(t, s) \in \Delta\}$ of linear operators on $X$, where $\Delta=\{(t, s): 0 \leq s \leq t \leq T\}$, which satisfies the following properties:

(E1) $U(t, r) U(r, s)=U(t, s)$ and $U(t, t)=I$ for $(t, r),(r, s) \in \Delta$.

(E2) $U(t, s)$ is strongly continuous on $\Delta$ into $B(X)$, and $\|U(t, s)\|_{X} \leq M$ for $(t, s) \in \Delta$.

(E3) $U(t, s)(Y) \subset Y$ for $(t, s) \in \Delta, U(t, s)$ is strongly continuous on $\Delta$ into $B(Y)$, and $\|U(t, s)\|_{Y} \leq M \exp \left(M \mid\|B\|_{Z}(t-s)\right)$ for $(t, s) \in \Delta$. 
(E4) $(\partial / \partial t) U(t, s) y=A(t) U(t, s) y$ for $(t, s) \in \Delta$ and $y \in Y$.

(E5) The integral equality

$$
S U(t, s) y=\Xi U(t, s) S y+\int_{s}^{t} \Xi U(t, r) B(r) S U(r, s) y d r
$$

holds for $(t, s) \in \Delta$ and $y \in Y$.

\section{Construction of approximate solutions}

This section is devoted to the construction of approximate solutions for (CP).

Let $\Delta_{0}=\left\{(t, s): 0 \leq t-s \leq \varepsilon_{0}, 0 \leq s \leq t \leq T\right\}$. For simplicity of notation, we write $J(t, s)$ instead of $(I-(t-s) A(t))^{-1}$ for $(t, s) \in \Delta_{0}$.

Lemma 1. Let $\left\{\sigma_{i}\right\}_{i=0}^{l}$ be a sequence such that $0 \leq \sigma_{0}<\sigma_{1}<\cdots<\sigma_{l} \leq T$ and $\sigma_{i}-\sigma_{i-1} \leq \varepsilon_{0}$ for $i=1,2, \ldots$, l. Let $x_{0} \in Y$ and set $x_{i}=\prod_{j=1}^{i} J\left(\sigma_{j}, \sigma_{j-1}\right) x_{0}$ for $1 \leq i \leq l$. Then we have, for $1 \leq i \leq l$,

(i) $x_{i} \in Y$ and

$$
S x_{i}=\prod_{j=1}^{i} \Xi J\left(\sigma_{j}, \sigma_{j-1}\right) S x_{0}+\sum_{j=1}^{i}\left(\sigma_{j}-\sigma_{j-1}\right) \prod_{l=j}^{i} \Xi J\left(\sigma_{l}, \sigma_{l-1}\right) B\left(\sigma_{j}\right) S x_{j},
$$

(ii) $\left\|S x_{i}\right\|_{Z} \leq M \exp \left(2 M\|\| B \|_{Z}\left(\sigma_{i}-\sigma_{0}\right)\right)\left\|S x_{0}\right\|_{Z}$.

Remark 2. Combination of (ii) and (A1) gives the estimate

$$
\left\|\prod_{j=1}^{i} J\left(\sigma_{j}, \sigma_{j-1}\right)\right\|_{Y} \leq M \exp \left(2 M\|\| B\|\|_{Z}\left(\sigma_{i}-\sigma_{0}\right)\right)
$$

for $1 \leq i \leq l$.

Proof. Assume that $x_{i-1} \in Y$ for some $i \in\{1,2, \ldots, l\}$. Then we have, by (A3), $x_{i} \in Y$ and

$$
S x_{i}=\Xi J\left(\sigma_{i}, \sigma_{i-1}\right)\left(S x_{i-1}+\left(\sigma_{i}-\sigma_{i-1}\right) B\left(\sigma_{i}\right) S x_{i}\right) .
$$

The desired claim (i) is proved by induction.

To prove (ii), we estimate (1) to obtain the inequality

$$
\left\|S x_{i}\right\|_{Z} \leq M\left\|S x_{0}\right\|_{Z}+\sum_{j=1}^{i}\left(\sigma_{j}-\sigma_{j-1}\right) M\|\| B\|\|_{Z}\left\|S x_{j}\right\|_{Z}
$$

for $1 \leq i \leq l$. By $b_{i}$ we denote the right-hand side of the inequality above. Then we have $\left\|S x_{i}\right\|_{Z} \leq b_{i}$ and $b_{i}-b_{i-1} \leq\left(\sigma_{i}-\sigma_{i-1}\right) M\|\| B \|_{Z} b_{i}$. Solving this inequality we obtain the desired estimate (ii).

Lemma 2. The following assertions hold:

(i) The function $(t, s) \rightarrow \Xi J(t, s)$ is strongly continuous on $\Delta_{0}$ into $B(Z)$.

(ii) The function $(t, s) \rightarrow J(t, s)$ is strongly continuous on $\Delta_{0}$ into $B(Y)$.

Proof. Let $\left(t_{0}, s_{0}\right),(t, s) \in \Delta_{0}$ and $y \in Y$. By condition (A3) we have $J\left(t_{0}, s_{0}\right) y \in Y$ and

$$
S J\left(t_{0}, s_{0}\right) y=\Xi J\left(t_{0}, s_{0}\right) S y+\left(t_{0}-s_{0}\right) \Xi J\left(t_{0}, s_{0}\right) B\left(t_{0}\right) S J\left(t_{0}, s_{0}\right) y .
$$


Since $J(t, s) y-J\left(t_{0}, s_{0}\right) y=J(t, s)\left\{(t-s) A(t)-\left(t_{0}-s_{0}\right) A\left(t_{0}\right)\right\} J\left(t_{0}, s_{0}\right) y$, it follows from conditions (A1) and (A2) that the function $(t, s) \rightarrow J(t, s)$ is strongly continuous on $\Delta_{0}$ into $B(X)$. This fact together with the strong continuity of $\Xi$ implies that assertion (i) is true.

The desired assertion (ii) follows from the fact (i) and the inequality

$$
\begin{aligned}
& \left\|S J(t, s) y-S J\left(t_{0}, s_{0}\right) y\right\|_{Z} \\
& \quad \leq\left\|\Xi J(t, s) S y-\Xi J\left(t_{0}, s_{0}\right) S y\right\|_{Z} \\
& \quad+\left\|\left\{(t-s) \Xi J(t, s) B(t)-\left(t_{0}-s_{0}\right) \Xi J\left(t_{0}, s_{0}\right) B\left(t_{0}\right)\right\} S J\left(t_{0}, s_{0}\right) y\right\|_{Z} \\
& \quad+(t-s) M\|B\|\left\|_{Z}\right\| S J(t, s) y-S J\left(t_{0}, s_{0}\right) y \|_{Z}
\end{aligned}
$$

which is obtained by using the equality (2).

Lemma 3. Let $\left\{t_{i}\right\}_{i=0}^{\infty}$ be a sequence such that $0 \leq t_{0}<t_{1}<\cdots<t_{i}<\cdots \leq T$, $t_{i}-t_{i-1} \leq \varepsilon_{0}$ for $i \geq 1$ and $\lim _{i \rightarrow \infty} t_{i}=\bar{t}$. Then we have:

(i) The limit $\lim _{i \rightarrow \infty} \prod_{l=k+1}^{i} \Xi J\left(t_{l}, t_{l-1}\right) z$ exists in $Z$, for all $z \in Z$ and $k \geq 0$.

(ii) The limit $\lim _{i \rightarrow \infty} \prod_{l=1}^{i} J\left(t_{l}, t_{l-1}\right) y$ exists in $Y$, for all $y \in Y$.

Proof. Let $y \in Y$ and $k \geq 0$. For $i \geq j \geq k$ we have

$$
\begin{aligned}
& \prod_{l=k+1}^{i} J\left(t_{l}, t_{l-1}\right) y-\prod_{l=k+1}^{j} J\left(t_{l}, t_{l-1}\right) y \\
& \quad=\sum_{p=j+1}^{i}\left(I-\left(I-\left(t_{p}-t_{p-1}\right) A\left(t_{p}\right)\right)\right) \prod_{l=k+1}^{p} J\left(t_{l}, t_{l-1}\right) y
\end{aligned}
$$

and the right-hand side is estimated by $\left(t_{i}-t_{j}\right)\|\| A\left\|\left.\right|_{Y, X} M \exp \left(2 M\|\| B \|_{Z} T\right)\right\| y \|_{Y}$. Here we have used the estimate in Remark 2. Condition (A1) and the density of

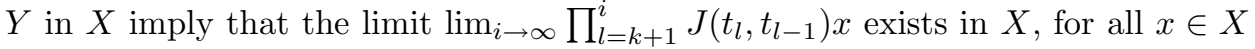
and $k \geq 0$. The desired claim (i) follows readily from the strong continuity of $\Xi$.

To prove (ii), put $x_{0}=\prod_{l=1}^{k} J\left(t_{l}, t_{l-1}\right) y$. Since $y \in Y$, we have $x_{0} \in Y$ by Lemma 1. Let $i \geq k$. By setting $\sigma_{l}=t_{l+k}$ for $l=0,1, \ldots, i-k, \prod_{l=1}^{i} J\left(t_{l}, t_{l-1}\right) y$ is written as $\prod_{l=1}^{i-k} J\left(\sigma_{l}, \sigma_{l-1}\right) x_{0}$. We use Lemma 1 to find the equality

$$
\begin{aligned}
S \prod_{l=1}^{i} J\left(t_{l}, t_{l-1}\right) y= & \prod_{l=k+1}^{i} \Xi J\left(t_{l}, t_{l-1}\right) S x_{0} \\
& +\sum_{p=k+1}^{i}\left(t_{p}-t_{p-1}\right) \prod_{l=p}^{i} \Xi J\left(t_{l}, t_{l-1}\right) B\left(t_{p}\right) S \prod_{l=k+1}^{p} J\left(t_{l}, t_{l-1}\right) x_{0} .
\end{aligned}
$$

It follows that the norm in $Z$ of the difference between $S \prod_{l=1}^{i} J\left(t_{l}, t_{l-1}\right) y$ and $\prod_{l=k+1}^{i} \Xi J\left(t_{l}, t_{l-1}\right) S x_{0}$ is bounded by $\left(t_{i}-t_{k}\right) M^{2}\|\mid B\|\left\|_{Z} \exp \left(2 M\|\| B\|\|_{Z} T\right)\right\| S y \|_{Z}$. By this fact and assertion (i) we have

$$
\begin{aligned}
\limsup _{i, j \rightarrow \infty} \| S \prod_{l=1}^{i} J\left(t_{l}, t_{l-1}\right) y- & S \prod_{l=1}^{j} J\left(t_{l}, t_{l-1}\right) y \|_{Z} \\
& \leq 2\left(\bar{t}-t_{k}\right) M^{2}\|\mid B\|\left\|_{Z} \exp \left(2 M\|\mid B\| \|_{Z} T\right)\right\| S y \|_{Z}
\end{aligned}
$$


for all $k \geq 0$. The desired assertion (ii) is proved by passing to the limit as $k \rightarrow$ $\infty$.

The following establishes the existence of approximate solutions for $(\mathrm{CP})$.

Proposition 1. Let $(s, y) \in[0, T) \times Y$ and $\varepsilon \in\left(0, \varepsilon_{0}\right]$. Then there exists a sequence $\left\{\left(t_{i}, x_{i}\right)\right\}_{i=0}^{N}$ in $[s, T] \times Y$ which satisfies the following properties:

(i) $s=t_{0}<t_{1}<\cdots<t_{N}=T$.

(ii) $t_{i}-t_{i-1} \leq \varepsilon$ for $i=1,2, \ldots, N$.

(iii) $\left(x_{i}-x_{i-1}\right) /\left(t_{i}-t_{i-1}\right)=A\left(t_{i}\right) x_{i}$ for $i=1,2, \ldots, N$, where $x_{0}=y$.

(iv) $\left\|x_{i}-x_{i-1}\right\|_{Y} \leq \varepsilon$ for $i=1,2, \ldots, N$.

(v) $\left\|\left(A(t)-A\left(t_{i-1}\right)\right) x_{i-1}\right\|_{X} \leq \varepsilon$ for $t \in\left[t_{i-1}, t_{i}\right]$ and $i=1,2, \ldots, N$.

Proof. We set $\left(t_{0}, x_{0}\right)=(s, y)$, and assume that a sequence $\left\{\left(t_{i}, x_{i}\right)\right\}_{i=0}^{k-1}$ in $[s, T] \times Y$ has been defined so that properties (i) through (v) may hold for $0 \leq i \leq k-1$, where $k \geq 1$ is an integer. If $t_{k-1}=T$, then the proof is complete. If $t_{k-1}<T$, then we define $h_{k}$ by the largest number satisfying the following conditions:

$$
\left\{\begin{array}{l}
0 \leq h_{k} \leq \varepsilon, \text { and } t_{k-1}+h_{k} \leq T \\
\left\|\left(I-h_{k} A\left(t_{k-1}+h_{k}\right)\right)^{-1} x_{k-1}-x_{k-1}\right\|_{Y} \leq \varepsilon \\
\left\|\left(A(t)-A\left(t_{k-1}\right)\right) x_{k-1}\right\|_{X} \leq \varepsilon \text { for } t \in\left[t_{k-1}, t_{k-1}+h_{k}\right]
\end{array}\right.
$$

We note that $h_{k}>0$ by (ii) of Lemma 2 and the strong continuity of $A$. Now, we define $t_{k}=t_{k-1}+h_{k}$ and $x_{k}=\left(I-h_{k} A\left(t_{k}\right)\right)^{-1} x_{k-1}$. Clearly, $t_{k-1}<t_{k}$, and $x_{k} \in Y$ by Lemma 1. It remains to prove that there exists an integer $N$ such that $t_{N}=T$. Suppose, contrary to our claim, that $t_{i}<T$ for all $i \geq 0$, and put $\bar{t}=\lim _{i \rightarrow \infty} t_{i}$. Then we deduce from (ii) of Lemma 3 that the sequence $\left\{x_{i}\right\}_{i=0}^{\infty}$ converges to an element $\bar{x}$ in $Y$ as $i \rightarrow \infty$. For $i \geq 1$ we set $\gamma_{i}=\bar{t}-t_{i-1}$. Clearly, $h_{i}<\gamma_{i}$ and $t_{i-1}+\gamma_{i}=\bar{t} \leq T$ for all $i \geq 1$. Moreover, $\lim _{i \rightarrow \infty} \gamma_{i}=0$. By (ii) of Lemma 2 we see that $\left(I-\gamma_{i} A\left(t_{i-1}+\gamma_{i}\right)\right)^{-1} x_{i-1}=\left(I-\left(\bar{t}-t_{i-1}\right) A(\bar{t})\right)^{-1} x_{i-1}$ tends to $\bar{x}$ in $Y$ as $i \rightarrow \infty$. Therefore there exists an integer $i_{0} \geq 1$ such that $\gamma_{i} \leq \varepsilon$ and $\left\|\left(I-\gamma_{i} A\left(t_{i-1}+\gamma_{i}\right)\right)^{-1} x_{i-1}-x_{i-1}\right\|_{Y} \leq \varepsilon$ for $i \geq i_{0}$. By the definition of $h_{i}$, we see that for each $i \geq i_{0}$ there exists $\hat{t}_{i} \in\left[t_{i-1}, t_{i-1}+\gamma_{i}\right]$ such that

$$
\left\|\left(A\left(\hat{t}_{i}\right)-A\left(t_{i-1}\right)\right) x_{i-1}\right\|_{X}>\varepsilon \text {. }
$$

Since $\bar{t}=\lim _{i \rightarrow \infty} \hat{t}_{i}$, the strong continuity of $A$ implies $\varepsilon \leq 0$, by taking the limit in the inequality above as $i \rightarrow \infty$. This contradicts the fact that $\varepsilon>0$.

\section{Generation of evolution operators}

This section is devoted to the proof of the Main Theorem which falls naturally into two parts. One is the proof of the uniqueness of the family $\{U(t, s):(t, s) \in$ $\Delta\}$ of linear operators satisfying properties (E1) through (E5), which is given by Theorem 1. The other is the proof of the existence of such a family, which is completed by combining Theorems 2 and 3 .

Let $P=\left\{0=s_{0}<s_{1}<\cdots<s_{K}=T\right\}$ be a partition of $[0, T]$ such that $s_{i}-s_{i-1} \leq \varepsilon_{0}$ for $i=1,2, \ldots, K$. Then, for such a partition $P$ of $[0, T]$ we define an operator $U(t, s ; P)$ on $\Delta_{0}$ by

$$
U(t, s ; P)=\prod_{l=p+1}^{i} J\left(s_{l}, s_{l-1}\right)
$$


whenever $t \in\left(s_{i-1}, s_{i}\right] \cap[0, T]$ and $s \in\left(s_{p-1}, s_{p}\right] \cap[0, T]$. Here and subsequently, we set $s_{-1}=-\infty$ for convenience. If $F$ is an operator-valued function on $[0, T]$, then we define a step function $F(t ; P)$ by

$$
F(t ; P)=F\left(s_{i}\right) \quad \text { for } t \in\left(s_{i-1}, s_{i}\right] \cap[0, T] \text { and } i=1,2, \ldots, K .
$$

The following is useful to prove that the $\operatorname{limit} \lim _{|P| \rightarrow 0} U(t, s ; P) x$ exists in $X$, for $x \in X$ and $(t, s) \in \Delta$.

Lemma 4. Let $(s, y) \in[0, T) \times Y$ and $\varepsilon \in\left(0, \varepsilon_{0}\right]$. Let $\left\{\left(t_{i}, x_{i}\right)\right\}_{i=0}^{N}$ be a sequence in $[s, T] \times Y$ as in Proposition 1 , and define a step function $u:[s, T] \rightarrow X$ by

$$
u(t)= \begin{cases}x_{0} & \text { for } t=s, \\ x_{i} & \text { for } t \in\left(t_{i-1}, t_{i}\right] \text { and } i=1,2, \ldots, N .\end{cases}
$$

If $P=\left\{0=s_{0}<s_{1}<\cdots<s_{K}=T\right\}$ is a partition of $[0, T]$ satisfying the condition

$$
|P|:=\max _{1 \leq k \leq K}\left(s_{k}-s_{k-1}\right) \leq \min _{1 \leq i \leq N}\left(t_{i}-t_{i-1}\right),
$$

then we have

$$
\|u(t)-U(t, s ; P) y\|_{X} \leq\left(1+M\left(1+2\left(1+\|\| A\|\|_{Y, X}\right) T\right)\right) \varepsilon
$$

for $t \in[s, T]$.

Proof. Since $P$ is a partition of $[0, T]$, there exists $p \in\{0,1, \ldots, K\}$ such that $t_{0}=$ $s \in\left(s_{p-1}, s_{p}\right] \cap[0, T]$. We note that $p \neq K$ by condition (5). For $k=p+1, \ldots, K$ we define $y_{k}=J\left(s_{k}, s_{k-1}\right) y_{k-1}$ and $y_{p}=x_{0}=y$. Moreover, we use an auxiliary function $v:[s, T] \rightarrow X$ defined by

$$
v(t)=x_{i-1}+\left(t-t_{i-1}\right) A\left(t_{i}\right) x_{i} \quad \text { for } t \in\left[t_{i-1}, t_{i}\right] \text { and } i=1,2, \ldots, N .
$$

Since $s_{p} \in\left[t_{0}, t_{1}\right]$, we have by (iii) and (iv) of Proposition 1 ,

$$
\left\|v\left(s_{p}\right)-y_{p}\right\|_{X}=\left(s_{p}-t_{0}\right)\left\|A\left(t_{1}\right) x_{1}\right\|_{X} \leq\left\|x_{1}-x_{0}\right\|_{Y} \leq \varepsilon .
$$

For $k=p+1, \ldots, K$, we set

$$
z_{k}=v\left(s_{k}\right)-v\left(s_{k-1}\right)-\left(s_{k}-s_{k-1}\right) A\left(s_{k}\right) v\left(s_{k}\right) .
$$

Since $v\left(s_{k}\right)-y_{k}=J\left(s_{k}, s_{k-1}\right)\left(v\left(s_{k-1}\right)-y_{k-1}+z_{k}\right)$, we have inductively

$$
v\left(s_{k}\right)-y_{k}=\prod_{l=p+1}^{k} J\left(s_{l}, s_{l-1}\right)\left(v\left(s_{p}\right)-y_{p}\right)+\sum_{j=p+1}^{k} \prod_{l=j}^{k} J\left(s_{l}, s_{l-1}\right) z_{j}
$$

for $k=p, \ldots, K$. We need to estimate $z_{k}$ for $k=p+1, \ldots, K$. Now, let $k \in$ $\{p+1, \ldots, K\}$. By condition (5), either (i) there exists $i \in\{1,2, \ldots, N\}$ such that $s_{k-1}, s_{k} \in\left[t_{i-1}, t_{i}\right]$, or else (ii) there exists $i \in\{1,2, \ldots, N-1\}$ such that $s_{k-1} \in\left[t_{i-1}, t_{i}\right]$ and $s_{k} \in\left[t_{i}, t_{i+1}\right]$. We begin by considering the case of (i). By the definition of $v$ we have $z_{k}=\left(s_{k}-s_{k-1}\right)\left(A\left(t_{i}\right) x_{i}-A\left(s_{k}\right) v\left(s_{k}\right)\right)$. Since

$$
\begin{aligned}
A\left(t_{i}\right) x_{i}-A\left(s_{k}\right) v\left(s_{k}\right)= & A\left(t_{i}\right)\left(x_{i}-x_{i-1}\right)+\left(A\left(t_{i}\right)-A\left(t_{i-1}\right)\right) x_{i-1} \\
& +\left(A\left(t_{i-1}\right)-A\left(s_{k}\right)\right) x_{i-1}+A\left(s_{k}\right)\left(x_{i-1}-v\left(s_{k}\right)\right)
\end{aligned}
$$

and

$$
x_{i-1}-v\left(s_{k}\right)=\left(t_{i-1}-s_{k}\right) A\left(t_{i}\right) x_{i}=\left(\left(t_{i-1}-s_{k}\right) /\left(t_{i}-t_{i-1}\right)\right)\left(x_{i}-x_{i-1}\right),
$$

we have, by (iv) and (v) of Proposition 1,

$$
\left\|z_{k}\right\|_{X} \leq 2\left(1+\||| A \mid\|_{Y, X}\right)\left(s_{k}-s_{k-1}\right) \varepsilon .
$$


In the case of (ii) we see that $z_{k}$ is written as $\left(v\left(s_{k}\right)-v\left(t_{i}\right)\right)+\left(v\left(t_{i}\right)-v\left(s_{k-1}\right)\right)-$ $\left(s_{k}-s_{k-1}\right) A\left(s_{k}\right) v\left(s_{k}\right)$ or

$$
\left(s_{k}-t_{i}\right)\left(A\left(t_{i+1}\right) x_{i+1}-A\left(s_{k}\right) v\left(s_{k}\right)\right)+\left(t_{i}-s_{k-1}\right)\left(A\left(t_{i}\right) x_{i}-A\left(s_{k}\right) v\left(s_{k}\right)\right) .
$$

An argument similar to that in the case of (i) shows that the estimate (10) is also valid in this case. We estimate the equality (8) by using the inequalities (7) and (10). This yields

$$
\left\|v\left(s_{k}\right)-y_{k}\right\|_{X} \leq\left(1+2\left(1+\|\| A \|_{Y, X}\right)\left(s_{k}-s_{p}\right)\right) M \varepsilon
$$

for $k=p, \ldots, K$.

Now, we turn to the proof of (6). Let $t \in(s, T]$. Then there exists $k \in$ $\{1,2, \ldots, K\}$ such that $t \in\left(s_{k-1}, s_{k}\right]$. Since $s \in\left(s_{p-1}, s_{p}\right]$, we have $U(t, s ; P) y=y_{k}$. If $s_{k} \in\left(t_{i-1}, t_{i}\right]$ for some $i \in\{1,2, \ldots, N\}$, then $u(t)=x_{i-1}$ or $x_{i}$. It follows from (9) that $\left\|v\left(s_{k}\right)-u(t)\right\|_{X} \leq \varepsilon$. The desired estimate (6) is obtained by combining this estimate and (11).

Theorem 1. Let $\{U(t, s):(t, s) \in \Delta\}$ be a family satisfying properties (E1) through (E4) of the Main Theorem. Then the representation

$$
U(t, s) x=\lim _{|P| \rightarrow 0} U(t, s ; P) x
$$

holds for $x \in X$ and $(t, s) \in \Delta$.

Proof. Let $P=\left\{0=s_{0}<s_{1}<\cdots<s_{K}=T\right\}$ be a partition of $[0, T]$ and $(t, s) \in \Delta$. Then there exist $p, k \in\{0,1, \ldots, K\}$ such that $s \in\left(t_{p-1}, t_{p}\right] \cap[0, T]$ and $t \in\left(s_{k-1}, s_{k}\right] \cap[0, T]$. Let $y \in Y$ and set

$$
w_{l}=\int_{s_{l-1}}^{s_{l}}\left(A(\sigma) U\left(\sigma, s_{p}\right) y-A\left(s_{l}\right) U\left(s_{l}, s_{p}\right) y\right) d \sigma
$$

for $l=p+1, \ldots, k$. By condition (E4) we have

$$
U\left(s_{l}, s_{p}\right) y-U\left(s_{l-1}, s_{p}\right) y=\left(s_{l}-s_{l-1}\right) A\left(s_{l}\right) U\left(s_{l}, s_{p}\right) y+w_{l}
$$

and hence

$$
U\left(s_{k}, s_{p}\right) y=U(t, s ; P) y+\sum_{l=p+1}^{k} \prod_{j=l}^{k} J\left(s_{j}, s_{j-1}\right) w_{l}
$$

for $l=p+1, \ldots, k$. The desired claim follows from condition (A1) and the fact that the function $(t, s) \rightarrow A(t) U(t, s) y$ is uniformly continuous on $\Delta$ in $X$ by condition (A2) and property (E3).

Theorem 2. For each $x \in X$, the limit $U(t, s) x=\lim _{|P| \rightarrow 0} U(t, s ; P) x$ exists uniformly for $(t, s) \in \Delta$. The family $\{U(t, s):(t, s) \in \Delta\}$ has properties (E1) and (E2) of the Main Theorem.

Proof. Let $(s, y) \in[0, T) \times Y$ and $\varepsilon \in\left(0, \varepsilon_{0}\right]$. Then there exists a sequence $\left\{\left(t_{i}, x_{i}\right)\right\}_{i=0}^{N}$ in $[s, T] \times Y$ satisfying properties (i) through (v) of Proposition 1 . We now set $\delta=\min _{1 \leq i \leq N}\left(t_{i}-t_{i-1}\right)$. If $P$ and $\widehat{P}$ are two partitions of $[0, T]$ such that $|P| \vee|\widehat{P}| \leq \delta$, then we have by Lemma 4

$$
\|U(t, s ; P) y-U(t, s ; \widehat{P}) y\|_{X} \leq 2\left(1+M\left(1+2\left(1+\|\| A\|\|_{Y, X}\right) T\right)\right) \varepsilon
$$

for $t \in[s, T]$. This implies that, for each $x \in X$ and $(t, s) \in \Delta$, the limit $U(t, s) x=$ $\lim _{|P| \rightarrow 0} U(t, s ; P) x$ exists in $X$. Property (E1) is satisfied, since $U(t, t ; P)=I$ and 
$U(t, r ; P) U(r, s ; P)=U(t, s ; P)$ for $(t, r),(r, s) \in \Delta$. The second half of (E2) is obtained by the fact that $\|U(t, s ; P)\|_{X} \leq M$ for $(t, s) \in \Delta$ (by (A1)). The uniform convergence and the first half of (E2) follow from the fact that for each $y \in Y$, the family $\{U(t, s ; P) y: P\}$ is pseudoequicontinuous on $\Delta$. This fact will be proved as follows: Let $y \in Y$ and $P=\left\{0=s_{0}<s_{1}<\cdots<s_{K}\right\}$ any partition of $[0, T]$. By (3) we find the inequality

$$
\|U(t, s ; P) y-U(\hat{t}, s ; P) y\|_{X} \leq M \exp \left(2 M \mid\|B\|_{Z} T\right)(|t-\hat{t}|+2|P|) \mid\|A\|\left\|_{Y, X}\right\| y \|_{Y}
$$

for $(t, s),(\hat{t}, s) \in \Delta$. For $(t, s),(t, \hat{s}) \in \Delta$, the inequality

$$
\|U(t, s ; P) y-U(t, \hat{s} ; P) y\|_{X} \leq M(|s-\hat{s}|+2|P|)\left|\|A \mid\|\left\|_{Y, X}\right\| y \|_{Y}\right.
$$

is obtained by estimating the equality

$$
\prod_{l=p+1}^{j} J\left(s_{l}, s_{l-1}\right) y-\prod_{l=q+1}^{j} J\left(s_{l}, s_{l-1}\right) y=-\sum_{k=q+1}^{p} \prod_{l=k}^{j} J\left(s_{l}, s_{l-1}\right)\left(s_{k}-s_{k-1}\right) A\left(s_{k}\right) y
$$

for $j \geq p \geq q$.

Theorem 3. Let $\{U(t, s):(t, s) \in \Delta\}$ be the family obtained in Theorem 2. Then for each $y \in Y$ and $(t, s) \in \Delta, \lim _{|P| \rightarrow 0} U(t, s ; P) y=U(t, s) y$ in $Y$. Moreover, properties (E3) through (E5) of the Main Theorem are satisfied.

Proof. Let $y \in Y$. By a fixed point argument one verifies that there exists a unique $V \in C_{*}(\Delta: B(Y, Z))$ satisfying the Volterra-type integral equation

$$
V(t, s) y=\Xi U(t, s) S y+\int_{s}^{t} \Xi U(t, \sigma) B(\sigma) V(\sigma, s) y d \sigma
$$

for $(t, s) \in \Delta$. On the other hand, we find by (1)

$$
\begin{aligned}
S U(t, s ; P) y= & \Xi U(t, s ; P) S y \\
& +\sum_{p=k+1}^{i} \int_{s_{p-1}}^{s_{p}} \Xi U\left(t, s_{p-1} ; P\right) B(\sigma ; P) S U(\sigma, s ; P) y d \sigma
\end{aligned}
$$

for $t \in\left(s_{i-1}, s_{i}\right] \cap[0, T]$ and $s \in\left(s_{k-1}, s_{k}\right] \cap[0, T]$ where $i \geq k \geq 0$. Now, we set $\varphi(t, s)=\lim \sup _{|P| \rightarrow 0}\|S U(t, s ; P) y-V(t, s) y\|_{Z}$ for $(t, s) \in \Delta$. (Note here that $\|S U(t, s ; P) y\|_{Z} \leq M \exp \left(2 M\|\| B\|\|_{Z} T\right)\|S y\|_{Z}$ by Lemma 1.) Subtracting (12) from this, and estimating the resultant equality we obtain the inequality

$$
\varphi(t, s) \leq M \mid\|B\|_{Z} \int_{s}^{t} \varphi(\sigma, s) d \sigma
$$

for $(t, s) \in \Delta$. Here we have used that fact that, for $z \in Z, \lim _{|P| \rightarrow 0} \Xi U(t, s ; P) z=$ $\Xi U(t, s) z$ in $Z$ uniformly for $(t, s) \in \Delta$, which follows from the first part of Theorem 2 and the strong continuity of $\Xi$. Application of Gronwall's inequality gives $\varphi(t, s)=$ 0 for $(t, s) \in \Delta$. This means that the first part of the theorem is true and that all the other properties of (E3) except the estimate of $U(t, s)$ in the $B(Y)$ norm and (E5) are satisfied. Estimating the integral equality in (E5) and applying Gronwall's inequality we obtain $\|S U(t, s) y\|_{Z} \leq M \exp \left(M \mid\|B\|_{Z}(t-s)\right)\|S y\|_{Z}$ for $(t, s) \in \Delta$ and $y \in Y$; hence $\|U(t, s)\|_{Y} \leq M \exp \left(M\|\| B\|\|_{Z}(t-s)\right)$ for $(t, s) \in \Delta$. Property 
(E4) is proved by taking the limit in the following equality deduced from (3) as $|P| \rightarrow 0$ :

$$
U(t, s ; P) y-U(\hat{t}, s ; P) y=\int_{s_{j}}^{s_{i}} A(\sigma ; P) U(\sigma, s ; P) y d \sigma
$$

for $t \in\left(s_{i-1}, s_{i}\right] \cap[0, T], \hat{t} \in\left(s_{j-1}, s_{j}\right] \cap[0, T], s \in\left(s_{k-1}, s_{k}\right] \cap[0, T]$ where $i \geq j \geq k$, and $y \in Y$.

\section{REFERENCES}

1. T. Kato, Linear evolution equations of "hyperbolic" type, J. Fac. Sci. Univ. Tokyo 17 (1970), 241-258. MR 43:5347

2. T. Kato, Abstract evolution equations, linear and quasilinear, revisited, Lecture Notes in Math. 1540 (1993), 103-125. MR 95m:34108

3. K. Kobayasi, On a theorem for linear evolution equations of hyperbolic type, J. Math. Soc. Japan 31 (1979), 647-654. MR 81a:34051

4. K. Kobayasi and N. Sanekata, A method of iterations for quasi-linear evolution equations in nonreflexive Banach spaces, Hiroshima Math J. 19 (1989), 521-540. MR 91a:34048

Department of Mathematics, Faculty of Science, Okayama University, Okayama 7008530, JAPAN

E-mail address: tanaka@math.okayama-u.ac.jp 